\title{
Fetal mortality associated with cholestasis of pregnancy and the potential benefit of therapy with ursodeoxycholic acid
}

\author{
M H Davies, R C M A da Silva, S R Jones, J B Weaver, E Elias
}

\begin{abstract}
Cholestasis of pregnancy is associated with increased fetal morbidity and mortality and should be treated actively. The significance attached to pruritus in pregnancy is often minimal, but it is a cardinal symptom of cholestasis of pregnancy, which may have no other clinical features. Eight women with previous cholestasis of pregnancy were referred to The Liver Unit within a 12 month period for advice concerning future pregnancies. Thirteen pregnancies had been affected by cholestasis of pregnancy and 12 had been treated expectantly with resultant perinatal morbidity or mortality in 11 (one normal delivery), including; eight stillbirths, two premature deliveries with fetal distress (one died in perinatal period), and an emergency caesarean section for fetal distress. The other pregnancy was treated actively and delivery was uncomplicated. Subsequently, three of these cases with recurrent cholestasis of pregnancy were referred while pregnant. In each, cholestasis developed with severe pruritus, gross increase of serum bile acids, and deranged liver tests. Each was treated with the choleretic agent ursodeoxycholic acid, with rapid clinical improvement and resolution of deranged biochemistry. In conclusion, cholestasis of pregnancy continues to be treated expectantly despite its association with increased morbidity and mortality and evidence suggesting improved prognosis with active treatment and the potential of reducing the associated perinatal mortality. In an uncontrolled series of three patients with cholestasis of pregnancy, ursodeoxycholic acid seemed to provide safe and effective therapy.

(Gut 1995; 37: 580-584)
\end{abstract}

Keywords: cholestasis of pregnancy, fetal mortality, prognosis, treatment, ursodeoxycholic acid.

Cholestasis of pregnancy is a disease of unknown aetiology, which may cause severe and intractable pruritus. The maternal prognosis is excellent, with symptoms and biochemical derangements resolving rapidly post partum, but cholestasis of pregnancy is associated with significant fetal morbidity and mortality. ${ }^{1-9}$ Cholestasis of pregnancy typically develops during the third trimester of pregnancy, but occasionally presents before 20 weeks. By definition, dermatological disease should be absent, if a diagnosis is made on clinical criteria alone, ${ }^{9}$ but this is not necessary if biochemical evidence of cholestasis is obtained. Most patients are not jaundiced and many have normal standard liver function tests. Serum alanine transaminase (ALT) is the most sensitive of the conventional liver function tests, ${ }^{10-12}$ although serum bile acids are invariably increased. 101113 Symptoms vary from mild pruritus to intractable pruritus, associated with gastrointestinal upset and icterus. Symptoms almost invariably resolve within one week of delivery.

Treatment of cholestasis of pregnancy varies widely. Despite evidence to the contrary, some obstetricians still perceive cholestasis of pregnancy as a benign condition and adopt an expectant policy. We report eight cases with a history of cholestasis of pregnancy whose pregnancies had mostly been treated conservatively. Three of these cases were pregnant at the time of referral and treated with the choleretic ursodeoxycholic acid.

Case reports and previous obstetric histories (Table)

\section{Case 1: 34 year old white woman}

First pregnancy, 1986 - pruritus from 28 weeks gestation. She was reassured. Spontaneous onset of labour at 37/40. Stillborn female. Pruritus settled within two days.

Second pregnancy 1988 - pruritus from $27 / 40$. Induction of labour at $38 / 40$, because of previous intra-uterine death. No meconium staining. Male baby, alive and well. Pruritus settled within four days.

Third pregnancy - pruritus from 26 weeks, without jaundice. Fetal movements were normal and she was reassured. Liver functions tests became abnormal, with increased serum aspartate transaminase (AST) (135). Spontaneous onset of labour at 39/40, with stillborn female. Pruritus settled almost immediately. 


\begin{tabular}{|c|c|c|c|c|c|c|c|c|c|c|c|c|}
\hline Case & Pregnancy & Onset & $\begin{array}{l}U D C A \\
R X\end{array}$ & Icterus & $\begin{array}{l}\text { A/N } \\
\text { clinic }\end{array}$ & $\begin{array}{l}\text { Onset } \\
\text { labour }\end{array}$ & Gestation & $\begin{array}{l}\text { Mode of } \\
\text { delivery }\end{array}$ & $A S T$ & $\begin{array}{l}\text { Meconium } \\
\text { staining }\end{array}$ & Outcome & $\begin{array}{l}\text { Cessation } \\
\text { of pruritus }\end{array}$ \\
\hline \multirow[t]{4}{*}{1} & 1st & $28 / 40$ & No & No & Yes & SPONT & $37 / 40$ & Vaginal & 243 & Yes & Stillbirth & 2 Days \\
\hline & 2nd & $27 / 40$ & No & No & Yes & Induced & $38 / 40$ & Vaginal & 129 & No & Normal & 4 Days \\
\hline & 3rd & $26 / 40$ & No & No & Yes & SPONT & Term & Vaginal & 135 & Yes & Stillbirth & 1 Day \\
\hline & 4th & $25 / 40$ & Yes & No & Yes & $\mathrm{C} / \mathrm{s}$ & $35 / 40$ & ELEC C/s & 213 & No & Normal & 2 Days \\
\hline \multirow[t]{3}{*}{2} & 1 st & $35 / 40$ & No & No & Yes & SPONT & $38 / 40$ & EMERG C/s & N/D & Yes & Fetal distress & 3 Days \\
\hline & 2nd & $35 / 40$ & No & No & Yes & SPONT & Term & Vaginal & 82 & Yes & Stillbirth & 1 Day \\
\hline & 3rd & $28 / 40$ & Yes & No & Yes & $\mathrm{C} / \mathrm{s}$ & $35 / 40$ & ELEC C/s & 42 & No & Normal & 0 Days \\
\hline \multirow[t]{4}{*}{3} & $1 \mathrm{st}$ & $28 / 40$ & No & No & Yes & SPONT & $36 / 40$ & Vaginal & N/D & $\mathrm{N} / \mathrm{K}$ & Perinatal death & 7 Days \\
\hline & 2nd & None & No & No & Yes & SPONT & Term & Vaginal & N/D & No & Normal & N/A \\
\hline & 3 rd & $32 / 40$ & No & No & Yes & SPONT & $37 / 40$ & Vaginal & N/D & No & Normal & 7 Days \\
\hline & 4th & $30 / 40$ & Yes & No & Yes & Induced & $38 / 40$ & Vaginal & 205 & No & Normal & 2 Days \\
\hline \multirow[t]{3}{*}{4} & $1 \mathrm{st}$ & None & No & N/A & Yes & SPONT & Term & Vaginal & N/D & No & Normal & N/A \\
\hline & 2nd & $30 / 40$ & No & No & Yes & SPONT & $36 / 40$ & Vaginal & N/D & $?$ & Fetal distress & 1 Week \\
\hline & 3 rd & $31 / 40$ & No & No & Yes & SPONT & Term & Vaginal & N/D & Yes & Stillbirth & 2 Days \\
\hline \multirow[t]{3}{*}{5} & $1 \mathrm{st}$ & None & No & N/A & Yes & SPONT & $35 / 40$ & Vaginal & N/D & Yes & PREM + NIL & $\mathrm{N} / \mathrm{A}^{\star}$ \\
\hline & 2nd & None & No & N/A & Yes & SPONT & Term & Vaginal & N/D & No & Normal & N/A \\
\hline & 3 rd & $25 / 40$ & No & No & Yes & SPONT & $32 / 40$ & Vaginal & N/D & Yes & Stillbirth & 1 Day \\
\hline 6 & $1 \mathrm{st}$ & $31 / 40$ & No & No & Yes & SPONT & Term & Vaginal & N/D & Yes & Stillbirth & 7 Days \\
\hline 7 & $1 \mathrm{st}$ & $28 / 40$ & No & No & Yes & SPONT & $37 / 40$ & Vaginal & $\mathrm{N} / \mathrm{D}$ & Yes & Stillbirth & 7 Days \\
\hline 8 & 1st & $32 / 40$ & No & No & Yes & SPONT & $37 / 40$ & Vaginal & 172 & Yes & Stillbirth & 1 Day \\
\hline
\end{tabular}

A summary of details of all pregnancies of eight females referred subsequently to The Liver Unit. A total of 13 pregnancies were affected by cholestasis of pregnancy, resulting in two normal deliveries, two premature deliveries with fetal distress (1 perinatal death), one emergency caesarean section at 38 weeks for fetal distress, and eight stillbirths occurred. *First baby of case 5 was born premature, but with no other complications. UDCA RX=ursodeoxycholic acid therapy; $\mathrm{N} / \mathrm{D}=$ liver function tests not done before delivery; A/N CLINIC=antenatal clinic; N/A= not applicable; N/K=not known; SPONT=spontaneous; EMERG/ELEC $\mathrm{C} / \mathrm{s}=$ emergency or elective caesarean section.

Case 2: 29 year old white woman

First pregnancy - pruritus from 35/40. Spontaneous onset of labour, at $38^{+}$weeks gestation. Emergency caesarean section for fetal distress. Baby discharged alive and well. Pruritus settled within three days.

Second pregnancy - pruritus from 35/40, without jaundice. Fetal movements were normal, she was reassured, and the pregnancy was treated routinely. Serum AST was increased at $82 \mathrm{IU} / 1$. Spontaneous onset of labour at term, with a stillborn baby delivered vaginally. Pruritus settled within one day.

\section{Case 3: 30 year old white woman}

First pregnancy - pruritus from 28 weeks. Spontaneous labour at $36 / 40$, with fetal distress. The baby was transferred to special care baby unit (SCBU). The baby was discharged home, but readmitted with recurrent convulsions and died in the perinatal period.

Second pregnancy - normal.

Third pregnancy - pruritus from 32/40. Spontaneous onset of labour at $37 / 40$, with delivery of healthy baby.

Case 4: 30 year old white woman

First pregnancy - normal.

Second pregnancy - pruritus from 30 weeks gestation. Early labour, at 36/40, with fetal distress. Baby discharged alive and well.

Third pregnancy - pruritus from 31 weeks gestation, without jaundice. The pregnancy was treated routinely. Liver function tests were not monitored and she was reassured. Spontaneous onset of labour at term, with stillborn baby. Pruritus settled within two days.

Case 5: 28 year old white woman

First pregnancy - normal pregnancy. Spontaneous labour 35/40, meconium stained liquor, baby discharged fit and well.

Second pregnancy - normal.
Third pregnancy - pruritus from 25 weeks gestation, without jaundice and liver function tests were not monitored. Fetal movements were normal and she was reassured. Onset of labour was spontaneous at 32 weeks, with a stillborn baby delivered vaginally. Pruritus settled within one day.

\section{Case 6: a 35 year old white woman}

First pregnancy - pruritus from 31 weeks, without jaundice. Fetal movements normal and the pregnancy was treated routinely. Liver function tests were not monitored. Spontaneous onset of labour at term, with a stillborn baby delivered vaginally. Pruritus settled within seven days.

\section{Case 7: a 28 year old white woman}

First pregnancy - pruritus from 28 weeks gestation, without jaundice. Liver function tests were not monitored and pregnancy was treated routinely. Spontaneous onset of labour at $37 / 40$, with a stillborn baby delivered vaginally. Pruritus settled within seven days.

Case 8: a 27 year old white woman

First pregnancy - pruritus from 32 weeks, without jaundice. Serum AST increased at 172 IU/l, but fetal movements were normal and she was reassured. Spontaneous labour at 37 weeks gestation, with a stillborn baby delivered vaginally. Relief of pruritus occurred immediately post partum.

\section{Cases treated with ursodeoxycholic acid}

Cases 1 to 3 were each referred during pregnancy. The clinical course, effect of ursodeoxycholic acid (UDCA) therapy, and outcome of these three pregnancies is described. Because UDCA is not licensed for use in cholestasis of pregnancy fully informed patient consent was obtained before this treatment. In case $1, \mathrm{~S}$ adenosyl-L-methionine was started before the 


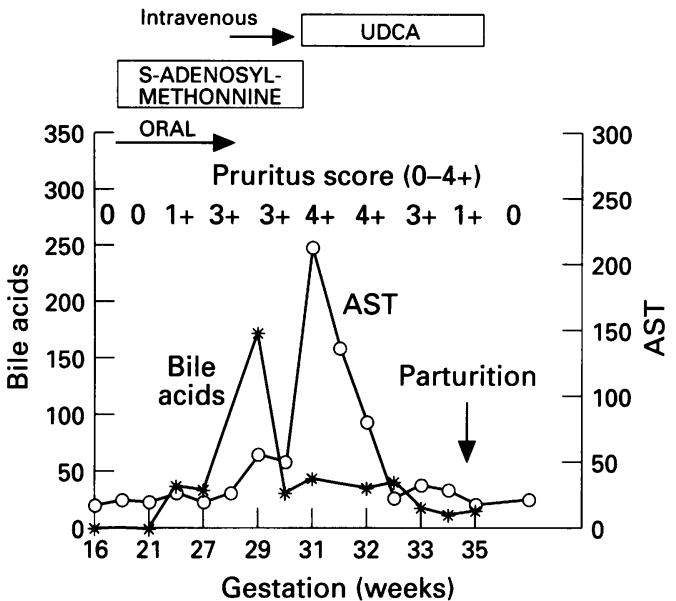

Figure 1: Clinical and biochemical parameters: case 1.

onset of symptoms in an effort to prevent cholestasis developing.

All three patients were booked to antenatal clinics at 10 weeks, while asymptomatic. Ultrasound scans at 16 weeks were normal in each.

Each received standard antenatal care. When symptoms of cholestasis developed inpatient observation was offered. Cardiotocography and Doppler ultrasound examinations of fetus and placenta were carried out weekly. In cases 1 and 2 , amniocentesis was performed at 35 weeks gestation to examine the lecithin:sphingomyelin ratio, to determine evidence of fetal lung maturity, and proceed to delivery by caesarean section, if appropriate. Labour was induced in case 3 at 38/40.

Each patient kept her own record of 'pruritus score' between 0 and $4+.0$ indicated no pruritus; $1+$ indicated occasional pruritus; $2+$ indicated more prolonged episodes; $3+$ indicated pruritus present more than absent; $4+$ represented almost constant pruritus, present by day and night.

Liver function tests were measured by the hospital clinical chemistry department. Samples for serum bile acids were stored at $-20^{\circ} \mathrm{C}$ and analysed using a commercial total serum bile acid kit, assaying $3 \alpha$-hydroxy bile acids (Sigma, UK).

Case 1 received S-adenosyl-L-methionine (Ademetionine), provided in oral and intravenous formulations by Knoll Ltd (BASF group).

Cases 1 and 2 received UDCA $750 \mathrm{mg}$ daily

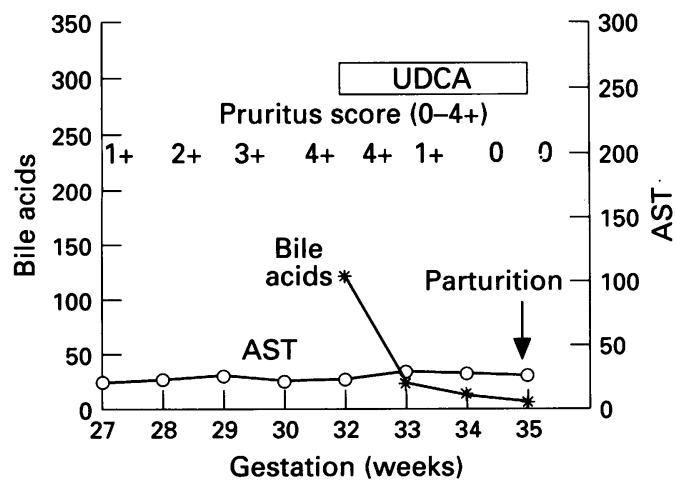

Figure 2: Clinical and biochemical parameters: case 2.
('Ursofalk', Thames Laboratories, UK). Case 3 received $1000 \mathrm{mg}$ daily.

\section{Progress}

Case 1

Figure 1 is a diagrammatic representation of serum AST, pruritus score, and serum bile acids.

The first trimester was normal. S-adenosylL-methionine $1600 \mathrm{mg}$ daily, was started orally at 16 weeks as prophylaxis, even though liver function tests and serum bile acids were normal. Dyspepsia and diarrhoea developed, so the dose was reduced to $1200 \mathrm{mg}$ daily. Despite S-adenosyl-L-methionine, pruritus developed at 25 weeks gestation. Liver function tests remained normal, but serum bile acid concentrations were increased. At 29 weeks, pruritus became increasingly severe and serum AST became abnormal. Intravenous S-adenosyl-L-methionine, $1200 \mathrm{mg}$ daily, was started, but AST continued to rise $-213 \mathrm{IU} / 1(<30 \mathrm{IU} / \mathrm{l})$ and pruritus became more troublesome. She was admitted to hospital for close monitoring. At 31 weeks, Sadenosyl-L-methionine was stopped and UDCA was started at $750 \mathrm{mg}$ daily, by mouth. Symptomatic improvement occurred within days and serum AST and serum bile acids fell to normal values within two weeks of starting treatment. Fetal monitoring by cardiotocography and Doppler ultrasound was satisfactory throughout this period.

Amniocentesis confirmed fetal lung maturity at 35 weeks gestation. Delivery of a liveborn male ( $35 / 40$ gestation) was performed by caesarean section. The baby was nursed on SCBU for nine days. He required oxygen therapy, but not mechanical ventilation. He was discharged home at 18 days.

\section{Case 2}

Figure 2 is a diagrammatic representation of liver function tests, pruritus score, and serum bile acids.

Liver function tests were initially normal. Pruritus developed at 28 weeks gestation and became severe. She was referred as an outpatient to The Liver Unit at 33 weeks gestation. Liver function tests were only mildly deranged, but serum bile acids were greatly increased. UDCA was started orally at $750 \mathrm{mg}$ daily. Pruritus completely resolved within five days of starting treatment, with return of serum bile acids to normal concentrations.

Amniocentesis was performed at 35 weeks to assess fetal lung maturity. The following day a liveborn female was delivered by caesarean section. She was nursed on SCBU for five days, requiring oxygen therapy, but not requiring assisted ventilation. She was discharged home at 10 days.

Case 3

Figure 3 is a diagrammatic representation of ALT, pruritus score, and serum bile acids. 


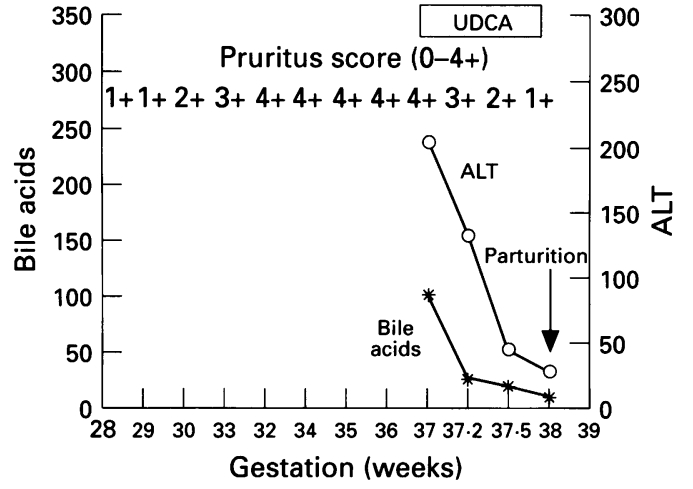

Figure 3: Clinical and biochemical parameters: case 3.

The first and second trimesters were normal. Pruritus developed at 30 weeks gestation. She was referred as an outpatient to The Liver Unit when liver function tests and serum bile acids were noted to be abnormal at 37 weeks. Treatment with UDCA was started orally at $1000 \mathrm{mg}$ daily. Serum ALT rapidly returned to normal from a peak of $205 \mathrm{IU} / 1$ (<30 IU/1). Serum bile acids rapidly declined from a peak of $102 \mathrm{U} / \mathrm{l}(<8 \cdot 1 \mathrm{U} / \mathrm{l})$ and pruritus, which had been intractable for several weeks had almost disappeared by the time she was induced at $38 / 40$. Serum bile acids responded rapidly.

A healthy female baby was delivered vaginally.

\section{Discussion}

Treatment of cholestasis of pregnancy varies widely. Despite evidence to the contrary, ${ }^{2-9} 12$ some obstetricians still perceive cholestasis of pregnancy as a benign condition and adopt an expectant treatment policy. We have reported eight cases who had experienced at least one previous episode of cholestasis of pregnancy and were referred to The Liver Unit within a 12 month period. All pregnancies, except one, had been treated conservatively receiving standard antenatal care, without additional fetal or maternal monitoring. All women were reassured that pruritus occurs within the spectrum of normal pregnancy and is 'nothing to worry about'. Each had experienced a stillbirth or perinatal death in association with cholestasis of pregnancy. One patient had suffered a second stillbirth and two had pregnancies complicated by fetal distress requiring immediate delivery. These were all associated with cholestasis of pregnancy.

When cholestasis of pregnancy was recognised as a clinical entity, it was considered benign. ${ }^{14-18}$ Subsequent reports showed a consistent pattern of increased fetal morbidity and mortality. ${ }^{2-9} 12$ Fifty six cases from Sydney had a perinatal death rate of $11 \%$, spontaneous premature labour in $36 \%$, and heavily meconium stained liquor in $27 \% .^{2}$ A later study from the same centre showed improved results, ${ }^{3}$ after an elective policy was instituted. Despite careful fetal monitoring, the authors were unable to predict a poor outcome in those babies who died. All stillbirths were associated with meconium staining. Amniocentesis for the presence/absence of meconium was the most sensitive predictor of fetal well being. ${ }^{3}$
A study of 18 patients from Vancouver ${ }^{1}$ recorded fetal distress in $33.3 \%$ and meconium staining in $58 \%$. These authors, ${ }^{1}$ and others ${ }^{3} 19$ recommended delivery when evidence of lung maturity has been established, although early delivery is not without morbidity. Shaw reported $59 \%$ morbidity in their intervention group. ${ }^{1}$

Some authors report fetal prognosis to correlate with disease severity. ${ }^{2820}$ In a prospective study of 117 patients with cholestasis of pregnancy, Laatikainen ${ }^{8}$ reported concentrations of serum bile acids to be a more sensitive predictor of fetal distress than standard liver function tests, so that women with highest serum bile acid concentrations should be subjected to more intensive monitoring. Even in mild or moderate cases, however, fetal risk was still noted. ${ }^{8}$ Biochemical markers of fetoplacental function, oestriol, and human placental lactogen, have proved ineffective at predicting fetal distress. ${ }^{21} 22$

Development of the optimal treatment policy in cholestasis of pregnancy is hindered by difficulty predicting fetal outcome. The stillborn babies in the cases we report showed no evidence of dysmaturity, suggesting that chronic nutritional deficiency was not present and all but one of the fetal deaths occurred at, or beyond, 37 weeks gestation. These findings are in accordance with previous reports, in which most stillbirths occurred late in pregnancy. ${ }^{2622-24}$ This provides a rationale for early delivery in cholestasis of pregnancy. Invasive means of monitoring may assist prognostication. ${ }^{3}$ Non-invasive measures should include regular cardiotocographs, yet these may remain normal until a very late stage, even in cases of stillbirth. ${ }^{8}$

Despite uncertainty as to the mechanism of cholestasis, treatment with choleretic agents has been shown to be of benefit in uncontrolled and controlled trials, including Sadenosyl-L-methionine, which has been used with variable benefit, ${ }^{25-28}$ although a double blind placebo controlled study showed no benefit. $^{29}$ Other agents used with apparent benefit include epomediol, ${ }^{30}$ dexamethasone, ${ }^{31}$ and UDCA. ${ }^{19} 32$

We have presented the progress of three women treated with UDCA, all of whom had previous stillbirths or perinatal deaths associated with cholestasis of pregnancy. Our cases suggest UDCA provides well tolerated and effective treatment. In each case there was rapid symptomatic relief and biochemical improvement. The mechanism of action of UDCA is unknown, but its effects probably result from modification of the bile acid pool, choleresis, and a reduction of serum bile acids. While there are concerns over prescribing novel drugs during pregnancy, the start of treatment is usually delayed until the third trimester, which minimises the risk of teratogenicity. There are no reports of adverse effects upon the fetus as yet.

Because the mechanism of fetal toxicity is unknown, it cannot be assumed that resolution of clinical and biochemical derangements associated with cholestasis of pregnancy will necessarily reduce fetal morbidity, though this seems a likely further benefit. 
UDCA has been of benefit in the treatment of cholestasis of pregnancy in other small series, ${ }^{19} 32$ but to date there are no results from controlled trials. Palma ${ }^{19}$ reported improvement of serum bile acid concentrations and pruritus in response to $1000 \mathrm{mg}$ UDCA daily in eight patients. In three of eight patients, withdrawal of treatment was associated with relapse of symptoms and biochemical derangement, which settled when UDCA was reinstituted. UDCA also reduces biochemical parameters of cholestasis in other cholestatic liver diseases. ${ }^{33} 34$

We have reported on eight women with cholestasis of pregnancy associated with eight stillbirths, a perinatal death, and two potential 'near misses'. We suggest there is a causal relation between cholestasis of pregnancy and the stillbirths, although this cannot be proved. Cholestasis of pregnancy causes distressing symptoms and is associated with significant increases in fetal morbidity and mortality. The aim of this report is to highlight the condition, which we consider to be commonly under recognised and inappropriately treated.

Its recognition is essential if early diagnosis is to permit timely fetal surveillance and intervention. Most stillbirths occur at or just before term. Elective delivery when fetal lung maturity is established could prevent the vast majority of such fetal loss. Appropriate obstetric treatment in cholestasis of pregnancy therefore represents a potential means by which perinatal mortality can be reduced.

UDCA, in a small, uncontrolled series seems effective at improving biochemical abnormalities and symptoms. Whether this would influence morbidity and mortality associated with the condition is unknown. These biochemical and clinical end points should be measured in controlled trials.

1 Shaw D, Frohlich J, Wittmann BAK, Willms M. A prospective study of 18 patients with cholestasis of pregnancy. $A m$ f Obstet Gynecol 1982; 142: 621-5.

2 Reid R, Ivey KJ, Rencoret RH, Storey B. Fetal complications of obstetric cholestasis. BMF 1976; 1: 870-2.

3 Fisk NM, Storey GNB. Fetal outcome in obstetric cholestasis. Br f Obstet Gynaecol 1988; 95: 1137-43.

4 Johnston WG, Baskett TF. Obstetric cholestasis. A 14 year review. Am ₹ Obstet Gynecol 1979; 133: 299-301.

5 Wilson BRI, Haverkamp AD. Cholestatic jaundice of pregnancy: new perspectives. Obstet Gynecol 1979; 54: pregnancy $650-2$.

6 Rencoret R, Aste H. Jaundice during pregnancy. Med f Aust 1973; 1: 167-9.

7 Roncaglia N, Trio D, Roffi L, Ciarli I, Tampieri A, Scian A, et al. Intrahepatic cholestasis of pregnancy: incidence, clinical course, complications. Ann Ostet Ginecol Med Perinat 1991; 112: 146-51.

8 Laatikainen T, Tulenheimo A. Maternal serum bile acid levels and fetal distress in cholestasis of pregnancy. Int $f$ Gynaecol Obstet 1984; 22: 91-4.
9 Berg B, Helm G, Petersohn L, Tryding N. Cholestasis of pregnancy. Clinical and laboratory studies. Acta Obste Gynecol Scand 1986; 65: 107-13.

10 Heikkinen J, Maentausta O, Ylostalo P, Janne O. Changes in serum bile acid concentrations during normal pregnancy, in patients with intrahepatic cholestasis of pregnancy and in pregnant women with itching. $\mathrm{Br} f$ Obstet Gynaecol 1981; 88: 240-5.

11 Laatikainen T, Ikonen E. Serum bile acids in cholestasis of pregnancy. Obstet Gynaecol 1977; 50: 313-8.

12 Fisk NM, Bye WB, Storey GNB. Maternal features of obstetric cholestasis: 20 years experience at King George V Hospital. Aust NZ F Obstet Gynaecol 1988; 28: 172-6.

13 Lunzer M, Barnes P, Byth K, O'Halloran M. Serum bile acid concentrations during pregnancy and their relationship to obstetric cholestasis. Gastroenterology 1986; 91: 825-9.

14 Sherlock S. In: Diseases of the liver and biliary system. Oxford: Blackwell Scientific, 1968: 296.

15 Barnes CG. In: Medical disorders in obstetric practice. Oxford: Blackwell Scientific, 1973: 160.

16 Davidson CS. Hepatic disease and pregnancy. $f$ Reprod Med 1973; 10: 107-10.

17 Orellana JM, Gonzalez AR, Rodrigues Y. Benign jaundice of pregnancy. Rev Med Chile 1961; 89: 676-9.

18 Roszkowski I, Miedzinska DP. Jaundice in pregnancy II Clinical course of pregnancy and delivery and condition of the neonate. Am $\mathcal{F}$ Obstet Gynecol 1968; 101: 500-3.

19 Palma J, Reyes H, Ribalta J, Iglesias J, Gonzalez MC Hernandez I, et al. Effects of ursodeoxycholic acid in patients with intrahepatic cholestasis of pregnancy. Hepatology 1992; 15: 1043-7.

20 Laatikainen T, Ikonen E. Fetal prognosis in obstetric hepatosis. Ann Chir Gynecol 1975; 64: 155-64.

21 Garoff L. Prediction of fetal outcome by urinary estriol, maternal serum placental lactogen and alpha-fetoprotein in diabetes and hepatosis of pregnancy. Obstet Gynecol in diabetes and $1976 ; 48: 659-66$.

22 Laatikainen TJ, Ikonen E. Serum bile acids in cholestasis of pregnancy. Ann Chir Gynaecol Fenn 1975; 64: 63

23 Friedlaender $\mathrm{P}$, Osler $\mathrm{M}$. Icterus and pregnancy. $A m \mathfrak{F}$ Obstet Gynecol 1967; 97: 894-900.

24 Ikonen E. Jaundice in late pregnancy. Acta Obstet Gynecol Scand 1964; 43 (suppl 5).

25 Stramentinoli G, Di Padova C, Gualano M, Rovagnati P Galli-Kienle $M$. Ethinylestradiol-induced impairment of bile secretion in the rat: protective effect of S-adenosyl-Lmethionine and its implication in estrogen metabolism. Gastroenterology 1981; 80: 154-8.

26 Boelsterli UA, Rakhit G, Balazs T. Modulation by S-adenosyl-L-methionine of hepatic $\mathrm{Na}^{+} \mathrm{K}^{+}$ATPase, membrane syl-L-methionine of hepatic $\mathrm{Na}^{+} \mathrm{K}^{+} \mathrm{ATPase}$, membrane
fluidity, and bile flow in rats with ethinyl estradiolfluidity, and bile flow in rats with ethinyl
induced cholestasis. Hepatology 1983; 3: 12-7.

27 Frezza M, Pozzato G, Chiesa L, Stramentinoli G, D Padova C. Reversal of intrahepatic cholestasis of pregnancy in women after high dose S-adenosyl-L-methionine administration. Hepatology 1984; 4: 274-8.

28 Bonfirraro G, Chieffi O, Quinti R, Tedesco R, LeGrazie C. S-adenosyl-L-methionine (SAMe)-induced amelioration of intrahepatic cholestasis of pregnancy: results of an open study. Drug Investigation 1990; 2: 125-8.

29 Ribalta J, Reyes H, Gonzalez MC, Iglesias J, Arrese M, Poniachik J. S-adenosyl-L-methionine in the treatment of patients with intrahepatic cholestasis of pregnancy: randomized, double-blind, placebo-controlled study with randomized, double-blind, placebo-controlled

30 Gonzalez MC, Iglesias J, Tiribelli C, Ribalta J, Reyes H, Hernandez I, et al. Epomediol ameliorates pruritus in patients with intrahepatic cholestasis of pregnancy. $\mathscr{f}$ Hepatol 1992; 16: 241-2.

31 Hirvioja M-L, Tuimala R, Vvori J. The treatment of intrahepatic cholestasis of pregnancy by dexamethasone. $B r \mathcal{F}$ Obstet Gynaecol 1992; 99: 109-1 1.

32 Mazella G, Rizzo N, Salzetta A, Iampieri R, Bovicelli L Roda E. Management of intrahepatic cholestasis in pregnancy. Lancet 1991; 338: 1594-5.

33 Leuschner U, Fischer H, Kurt W, Guldutuna S, Hubner $\mathrm{K}$, Hellstern $\mathrm{A}$, et al. Ursodeoxycholic acid in primary bil$\mathrm{K}$, Hellstern A, et al. Ursodeoxycholic acid in primary biliary cirrhosis: results of a controlled

34 Poupon ER, Eschwége E, Poupon R, UDCA-PBC Study Group. Ursodeoxycholic acid for treatment of primary biliary cirrhosis; interim analysis of a double-blind multicentre randomized trial. $\mathcal{F}$ Hepatol 1990; 11: 16-21. 